\title{
Simulation thermique de la fusion et de la recristallisation par lasers à excimères du silicium amorphe pour les applications transistors en couches minces
}

\author{
S. de Unamuno et E. Fogarassy
}

Laboratoire PHASE-CNRS, BP. 20, 67037 Strasbourg cedex, France

\begin{abstract}
Résumé: L'optimisation des technologies de fabrication de transistors en couches minces au silicium polycristallin par recuit laser à excimères, suppose une bonne connaissance des cinétiques de fusion et solidification rapides que l'on peut simuler grâce au développement d'un modèle thermique, basé sur la résolution de l'équation de la chaleur. Les simulations presentées ici sont réalisées pour différents lasers dans le domaine de la nanoseconde sur des couches de silicium amorphe deposées sur verre avec une couche tampon de $\mathrm{SiO}_{2}$.
\end{abstract}

Les transistors en couches minces (TFT) au silicium polycristallin [1] représentent un enjeu industriel important, tout particulièrement dans le domaine des écrans plats à matrice active.

La faisabilité des technologies assistées par lasers à excimères pour fabriquer, à basse température, des TFTs au $S i$ polycristallin présentant des mobilités en porteurs élevées (jusqu'à $640 \mathrm{~cm}^{2} / V . s$ pour le type $n$ et $400 \mathrm{~cm}^{2} / V . s$ pour le type $p$ [2]) et qui soient compatibles avec l'utilisation de substrats en verre bon marché, a été largement démontrée. [3]

L'optimisation du procédé nécessite, cependant, une meilleure connaissance de la cinétique de la fusion du silicium amorphe $(\mathrm{Si}-\mathrm{a})$ et de sa recristallisation rapide induite par une impulsion laser "nanoseconde", que l'on est capable de simuler en résolvant l'équation de la chaleur [4].

Nous nous sommes particulièrement intéressés à la distribution spatiale et temporelle de la température dans des multicouches $\mathrm{Si}-a / \mathrm{SiO}_{2}(300 \mathrm{~nm}) /$ verre, en fonction des conditions d'irradiation (fluence et forme temporelle de l'impulsion laser), d'une part avec un laser ArF à impulsion "courte" de 20 ns (EMG-201 MSC de LAMBDA PHYSIK) d'autre part avec un laser $\mathrm{XeCl}$ à impulsion "longue" de 150ns (VEL de la SOPRA).

La Figure 1 permet d'évaluer le seuil et la profondeur de fusion dans $S i-a$ en fonction de l'épaisseur du film amorphe et de la densité d'énergie pour les deux sources d'excimères considerées. On a ainsi la possibilité d'optimiser le traitement laser qui consiste à fondre la totalité de la couche de silicium tout en limitant les effets thermiques susceptibles de dégrader l'oxyde sous-jacent.

La durée de l'impulsion laser joue un rôle important sur la dynamique de fusion-solidification comme le montrent les simulations de la Figure 2. On observe, en particulier avec le laser VEL une diminution d'un facteur deux de la vitesse de recristallisation de la zone fondue ce qui peut avoir des conséquences favorables sur la morphologie et la taille des cristallites de $S i$ formées et donc sur la mobilité des porteurs dans la couche active du TFT.

On aboutit à une evolution comparable (réduction de la vitesse de solidification) en réalisant le recuit laser sur un échantillon chauffé ( $400 \mathrm{C}$ ) comme le montrent les simulations de la Figure 3.

Enfin la forme temporelle (montée lente ou rapide) de l'impulsion laser peut jouer un rôle sur la profondeur fondue mais n'a pas d'influence significative sur la vitesse de recristallisation (Figure 4). 


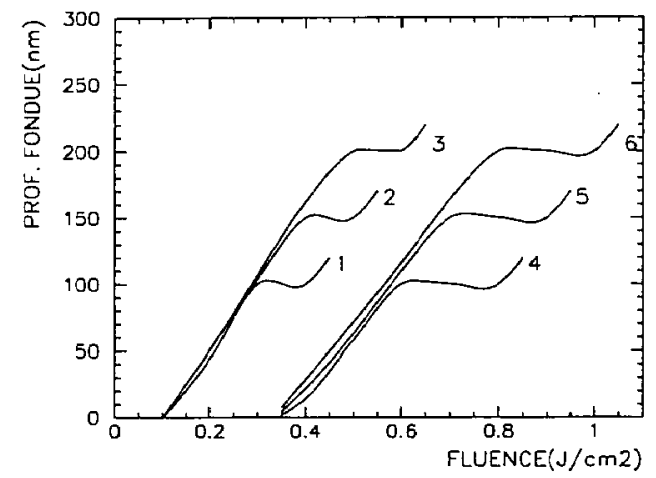

Fig 1: Profondeur fondue en fonction de la fluence pour 3 épaisseurs de $\mathrm{Si}-\mathrm{a}$ sur $300 \mathrm{~nm}$ de $\mathrm{SiO}_{2}$ sur Corning. (1) $100 \mathrm{~mm}$ de $S i-a, A r F$. (2) $150 \mathrm{~nm}$ de $S i-a$. ArF. (3) 200 umu de $S i-a$. ArF. (4) $100 \mathrm{~mm}$ de $S i-a$.VEL. (5) $150 \mathrm{~nm}$ de $S i-a$. VEL. (6) $200 \mathrm{~nm}$ de $S i-a$. VEL.

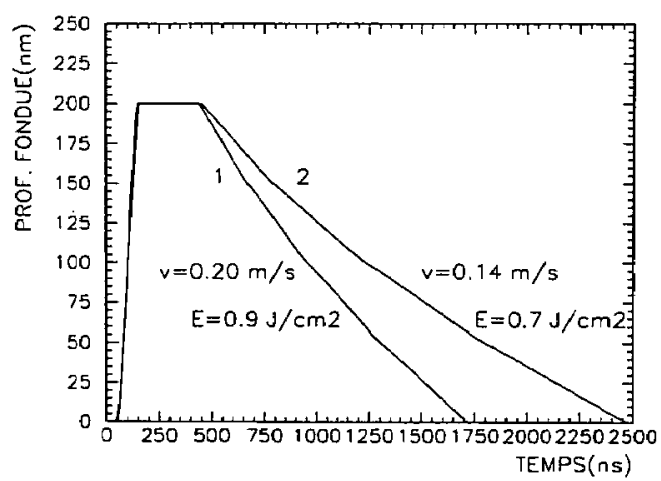

Fig 3: Dynamique du processus fusion-solidification. $200 \mathrm{~nm}$ le $\mathrm{Si}-a$ Sur 300 un de $\mathrm{SiO}_{2}$ sur Corning, lisser \EL. Influence de la températ ure initiale du substrat:

(1) $20 \mathrm{C}(2) 400 \mathrm{C}$.

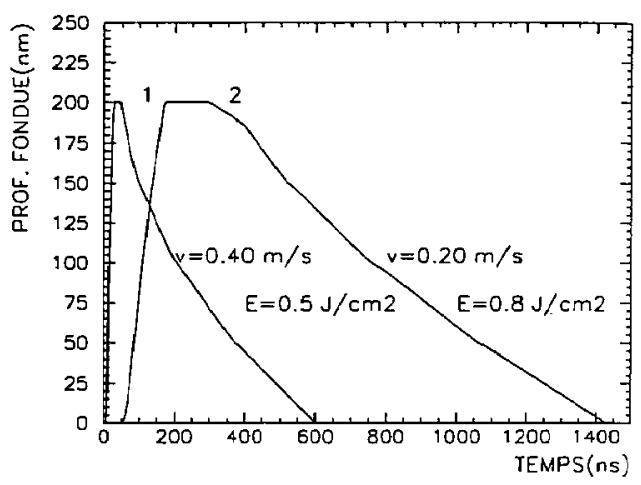

Fig 2: Dynamique du processus fusion-solidification $201 \mathrm{~mm}$ de $\mathrm{Si}-a$ sur $300 \mathrm{~nm}$ de $\mathrm{SiO}_{2}$ sur Corning (T initiale $=20$ ()). Inftuence de la clurée de l'inipulsion.

(1).ArF: $20 \mathrm{~ns}$. (2) VEL: $150 \mathrm{~ns}$.

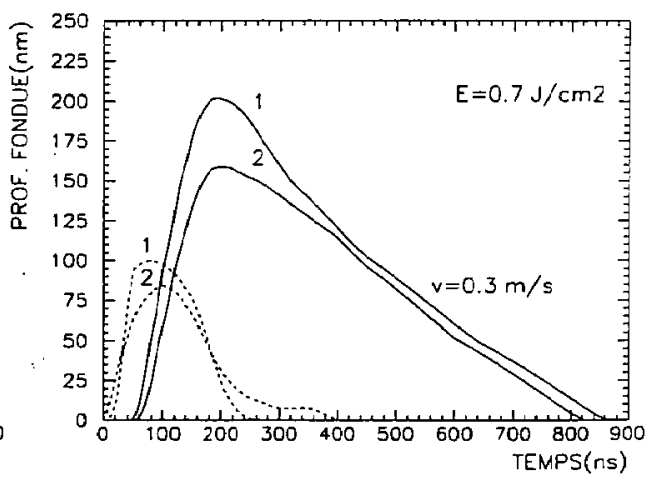

Fig 4: Dynamique du processus fusion-solidification. $200 \mathrm{~nm}$ cle $\mathrm{Si}-\mathrm{a}$ str $\mathrm{SiO}_{2}$. Influence de la forme du laser VEL. (en pointillé forme des impulsions laser(u.a.))

(1) Montée rapide. (2) Montée lente.

\section{Réfërences} 1994.

[1] "Ecrans plats pour visualisation" Revue Technique THOMSON-CSF Vol 26, $n^{\circ} 1$, Mars

[2] A. Kohno, T. Sameshima, N. Sano, M. Sekiya, M. Hara. IEEE Trans on Electron Dev,, Vol 42, $n^{\circ} 2$ (1995), 251.

[3] E. Fogarassy, M. Elliq, B. Prévot, F. Repplinger, A. Slaoui, R. Stuck, S. de Unamuno, E.L. Mathé et H. Pattyn. L.D. Laude(ed.), Excimer Lasers, 387-402, 1994 Kluwer Academic Publishers, Netherlands.

[4] S. de Unamuno and E. Fogarassy. Material Scïences and Engineering, B13 (1992) 29-33. 